\title{
Revision of the neotropical genus Sendaphne Nixon (Hymenoptera, Braconidae, Microgastrinae)
}

\author{
Jose L. Fernandez-Triana ${ }^{1,2}$, James B. Whitfield ${ }^{3}$, M. Alex Smith', \\ Winnie Hallwachs ${ }^{5}$, Daniel H. Janzen ${ }^{5}$
}

I Canadian National Collection of Insects, 960 Carling Ave., Ottawa, ON K1A OC6 Canada 2 Biodiversity Institute of Ontario, University of Guelph, Guelph, ON N1G 2W1 Canada 3 Department of Entomology, University of Illinois, Urbana, IL 61801 USA 4 Department of Integrative Biology, University of Guelph, Guelph, ON N1G 2W1 Canada 5 Department of Biology, University of Pennsylvania, Philadelphia, PA 19104-6018 USA

Corresponding author: Jose L. Fernandez-Triana (jftriana@uoguelph.ca)

Academic editor: G. Broad| Received 15 September 2014 | Accepted 9 December 2014 | Published 22 December 2014

http://zoobank.org/46F876D2-9FBD-4837-AB2C-A5FE64D8E468

Citation: Fernández-Triana JL, Whitfield JB, Smith MA, Hallwachs W, Janzen DH (2014) Revision of the neotropical genus Sendaphne Nixon (Hymenoptera, Braconidae, Microgastrinae) . Journal of Hymenoptera Research 41: 1-29. doi: 10.3897/JHR.41.8586

\begin{abstract}
The Neotropical genus of parasitoid wasps Sendaphne (Hymenoptera, Braconidae, Microgastrinae) is revised and the following six new species are described, all authored by Fernández-Triana and Whitfield: anitae, bennetti, broadi, dianariaspennae, penteadodiasae, and rogerblancoi. The greatest species richness is found in northern South America, but the genus extends north to $23^{\circ} \mathrm{N}$ in Mexico. Most species have been collected in rainforest below altitudes of $900 \mathrm{~m}$, with only a few species found in cloud forests up to $1900 \mathrm{~m}$. Nothing is known of the host caterpillars for these parasitoid wasps.
\end{abstract}

\section{Keywords}

Sendaphne, Microgastrinae, Neotropics, Area de Conservación Guanacaste, taxonomic revision, parasitoid wasps, DNA barcoding

Copyright Jose L. Fernandez-Triana et al. This is an open access article distributed under the terms of the Creative Commons Attribution License (CC BY 4.0), which permits unrestricted use, distribution, and reproduction in any medium, provided the original author and source are credited. 


\section{Introduction}

The genus Sendaphne was described by Nixon (1965) to accommodate two Neotropical species with a number of distinctive features: a very long and bilobate glossa, slender body, extensive yellow coloration, mostly smooth mesosoma and metasoma, enlarged hypopygium, and very long and curved ovipositor. Penteado-Dias (1995) described two additional species, and Scatolini and Penteado-Dias (1999) added another one. Four of the five species described so far are from Brazil, with the exception being Sendaphne sulmo Nixon (1965), recorded from southeastern Mexico. No host is known for Sendaphne, although the related genus Promicrogaster Brues \& Richardson, 1913, has been reared from hosts living in bracket fungi (Mason 1981), among other concealed hosts.

In spite of the rather unique morphological traits, the validity of Sendaphne as a distinct genus has been questioned even by the same author who described it (Nixon 1965: 204), and it might eventually be determined to be a synonym of the more abundant and diverse Promicrogaster. However, Mason (1981) considered the differences as sufficient to maintain them as two genera. That decision has been followed by all subsequent authors and, without a comprehensive phylogenetic study of Microgastrinae, we agree that is better to keep it that way at present. The morphological and molecular analysis of Whitfield et al. (2002), although not conclusive, also found those two genera to be closely related. An anticipated revision of Promicrogaster may help to elucidate the limits of these two genera in the future.

As part of comprehensive studies on the fauna of Microgastrinae from Area de Conservación Guanacaste (ACG), northwestern Costa Rica (e.g., Fernández-Triana et al. 2014 and references cited there) we found a new species from that area. The Canadian National Collection of Insects (CNC) in Ottawa, Canada and the NSF-funded "Insect Survey of a Hyperdiverse Country: Colombia" projects led by M. J. Sharkey, B. V. Brown and the Humboldt Institute (Colombia) also contained additional undescribed species from Central and South America. All these new species are described below, altogether with a key to all known, previously described species of Sendaphne.

\section{Methods}

Among Microgastrinae, Sendaphne is one of the most rarely collected genera, and is poorly represented in collections. This study is based on 73 specimens from four sources: 38 Neotropical specimens deposited in the CNC, 18 specimens from Colombia (Humboldt Institute), 10 specimens from the ACG inventory, four specimens from Unité d'Entomologie fonctionnelle et évolutive, Gembloux Agro-Bio Tech, Université de Liège (FUSAGx) and three specimens from the Natural History Museum, London, England (BMNH).

All species previously described were deposited in the Universidade Federal de São Carlos, São Carlos, São Paulo, Brazil (DCBU), Universidade Federal do Paraná, 
Curitiba, Paraná, Brazil (UFPR), or in the BMNH. We did not examine the holotypes of those species; however, their original descriptions and illustrations are sufficiently detailed to allow us to describe the new species with confidence.

Morphological terms and measurements of structures are mostly as used by Mason (1981), Huber and Sharkey (1993), Whitfield (1997), Karlsson and Ronquist (2012), and Fernández-Triana et al. (2014). Because the ovipositor in Sendaphne is strongly curved, its length was difficult to measure accurately; the ovipositor length measurements provided for each new species are only intended as an approximation. In any case, the ovipositor and its sheaths are some of the longest observed in any Microgastrinae genera; they are usually two times longer than the metatibia length.

Descriptions of the new species are based on the study of all available female specimens, so as to reflect intraspecific variation, but always include data from the holotype. As an exception, two new species only known from males are described below because they were sufficiently distinct to be distinguished from all others; the males of those two species may be identified by the key, but males of most other species may not be readily identified unless associated with females via rearing or molecular data.

The descriptions include 17 characters that are commonly used in describing Microgastrinae (e.g., body measurements such as length of body and fore wing, ovipositor sheath; and also color of particular body areas). Those descriptions are complemented with extensive color photos of every species. Geographic distribution is also provided in the key as supplementary information to aid the morphological identification of species, though we recognize that with time the current known geographic distribution may eventually become obsolete.

Photos were taken with a Keyence VHX-1000 Digital Microscope, using a lens with a range of 13-130x. Multiple images through the focal plane were taken of a structure and these were combined to produce a single in-focus image, using the software associated with the Keyence System.

Together with morphological studies, we also analyzed DNA barcodes (the 5 ' region of the cytochrome $c$ oxidase I (CO1) gene, Hebert et al. 2003) whenever available. DNA barcodes for all ACG inventory Sendaphne specimens were obtained using DNA extracts prepared from single legs using a glass fibre protocol (Ivanova et al. 2006). Briefly, total genomic DNA was re-suspended in $30 \mu \mathrm{l}$ of $\mathrm{dH} 2 \mathrm{O}$, and a 658-bp region near the 5' terminus of the $\mathrm{CO} 1$ gene was amplified using standard primers (LepF1-LepR1) following established protocols (Smith et al. 2006, 2007, 2008). If the initial $658 \mathrm{bp}$ amplification was unsuccessful, smaller sequences were generated using internal primers. If each amplification worked a composite sequence was generated, however in cases where only one read amplified, this shorter sequence was used. All information for the sequences associated with each individual specimen can be retrieved from the Barcode of Life Data System (BOLD) (Ratnasingham and Hebert 2007) using the following public DOI: http://dx.doi.org/10.5883/DSSENDAPH. A Neighbor-Joining tree based on Kimura 2-parameter distances of all described species of Sendaphne with DNA barcodes available in BOLD was also generated (Suppl. material 2). 


\section{Results}

Six new species of Sendaphne are described below, increasing the total known species from five to 11 . We are aware of potential additional new species in the $\mathrm{CNC}$ collection, from Costa Rica (but not ACG), Ecuador, and Brazil. However, they are not described here because each is only represented by a single specimen and they are not sufficiently distinct to warrant description without further specimens and evidence.

Sendaphne is a Neotropical genus. To date it is most abundant and diverse in South America (eight species), while Central America has three species. It extends from $23^{\circ} \mathrm{N}$ in central Mexico (Durango) to $27^{\circ} \mathrm{S}$ in Paraguay (Pirapo) and southern Brazil (Santa Catarina). Most of the species have been collected in rain forests, at altitudes between $100 \mathrm{~m}$ and $900 \mathrm{~m}$. However, a few species have been found only in cloud forests between $1,450 \mathrm{~m}$ and $1,900 \mathrm{~m}$. The specimens collected at higher altitudes have darker coloration (especially on mesosoma and metasoma) than those found in the lowlands.

An interesting result of our morphological study was the relation between body and fore wing lengths. Body proportions in Microgastrinae have not been explored in detail, but in most genera and species with available data the fore wing length tends to be slightly longer than the body length (usually by $0.1-0.2 \mathrm{~mm}$ ). In the specimens of Sendaphne described here, the body length was longer than the fore wing length (usually by $0.2-0.4 \mathrm{~mm}$ ). The main reason is the long and slender body form (rather than exceptionally short wings), and an unusually enlarged and extended hypopygium.

\section{Observations of COI barcodes for Sendaphne}

Of 46 specimens sampled (Suppl. material 1), we recovered sequences for only 24 specimens. Only three sequences were over 600 base pairs, while the rest were mostly minibarcodes of 102-390 base pairs each. However, six out of the 11 known species now have some molecular data associated (Suppl. material 2). Many of the initial amplifications from ACG were characterised by unintended amplification of the endosymbiont bacteria Wolbachia that were amplified in the first round LepF1/LepR1. These sequences were not mistaken for the wasp DNA (Smith et al. 2012) and are retained on BOLD in the trace files. The standard strategy of amplifying two smaller and overlapping regions secondarily was then followed (as the first round was considered a 'failure'). In this case the 5' amplification (LepF1/C_ANTMR1D) worked and the 3' amplification (RonMWASPdeg_t1 - LepR1) failed. Thus many of the ACG Sendaphne are -260-280bp in length. Finally, regarding the CNC specimens, these were, on average, 35 years old. In those cases, greater success with smaller amplifications compared to larger amplifications is to be expected (Hajibabaei et al 2006). In 2010 and 2011 when these specimens were submitted to the Biodiversity Institute of Ontario (Guelph) for DNA barcoding, they only had the primers generating minibarcode (smaller amplicons) used for PCR. The longer amplicon generating primer pairs were not attempted on these specimens. 


\section{Genus Sendaphne}

Sendaphne Nixon, 1965: 203.

Diagnosis. Glossa elongate and bilobate (Figs 2, 9, 34, 48, 58, 70). Lateral face of scutellum with polished area (=lunules) occupying most of the lateral face (Figs 5, 12, $19,28,44,49,56)$. Propodeum usually smooth and without carina (exceptionally having sparse punctures and few rugae on the nucha) (Figs 7, 13, 19, 28, 33, 44, 49, $60,74)$. Metacoxa very long, about the same length as metafemur length and metatibia length (Figs 8, 13, 15, 29, 50, 54, 61, 68, 74). Mediotergite 1 strongly narrowing towards posterior margin (Figs 4, 14, 19, 50, 51). Mediotergite 2 subtriangular, much longer medially than its width at its anterior margin (and usually also longer medially than its width at posterior margin). Ovipositor very long for a microgastrine wasp (two times longer than metatibia length) and strongly curved (Figs 1, 20, 35, 53, $54,57,64,68$ ); apex of ovipositor usually not sinuate (exceptionally with very slight sinuation). Fore wing with very wide first discal cell, and with small areolet (Figs 3, 17, 17, 24, 39, 55, 62, 69) (areolet sometimes not well-defined because veins 3RSa and $\mathrm{r}-\mathrm{m}$ are spectral, as in Figs 10,31, 47). Body color often mostly yellow to orange (with a few exceptions from species collected at higher altitudes, which have head, mesosoma and parts of metasoma dark brown to black). Body length longer than the fore wing length, usually by $0.2-0.4 \mathrm{~mm}$. Within Microgastrinae, Sendaphne can only be confused with Promicrogaster, but the later has a more transverse mediotergite 2, apex of ovipositor clearly sinuate, and propodeum usually with more sculpture and carination present.

\section{Key to Sendaphne species}

[This key is intended for female specimens, although two species are only known from males, and in those cases the key accommodates them. Generally, males tend to have darker coloration than the females, especially on the metasoma].

$1 \quad$ Head and mesosoma entirely dark brown to black (Figs 23, 24, 27, 28, 37$44,68-75$ ), metasoma entirely dark brown (Figs 69, 74, 75) or at most with mediotergites 1-2 orange-yellow (as in Figs 24, 25, 28, 39, 44) [all specimens collected above $1,460 \mathrm{~m}$, in cloud forests].............................................

- Color variable but much lighter than previous couplet, if head entirely dark brown to black, then mesosoma entirely or mostly yellow, orange or reddishyellow, and metasoma at most with brown bands on posterior margins of mediotergites 3+ [all specimens collected below $900 \mathrm{~m}$, in rainforests] .......4

2(1) Tegula, metacoxa, and mediotergites 1-2 dark brown to black (Figs 69, 7375); body very slender, metasoma longer than combined length of head and mesosoma (Fig. 68) [Costa Rica] 
- Tegula, metacoxa and mediotergites 1-2 yellow to reddish-yellow (Figs 23-28, 37-40, 42-44); body less slender, metasoma shorter than combined length of head and mesosoma (Figs 23, 37) [Ecuador, Mexico] ...................3

3(2) Mediotergite 1 length $6.0 \times$ its width at posterior margin, mediotergite 2 length $1.5 \times$ its width at posterior margin (Figs 24, 25, 28); first discal cell $2.0 \times$ as wide as high (Fig. 24) [Ecuador]

Sendaphne broadi Fernández-Triana $\&$ Whitfield, sp. n.

Mediotergite 1 length $4.0 \times$ its width at posterior margin, mediotergite 2 length $1.0 \times$ its width at posterior margin (Figs 39, 42, 44); first discal cell $1.3 \times$ as wide as high (Fig. 39) [Mexico]

Sendaphne bennetti Fernández-Triana \& Whitfield, sp. n.

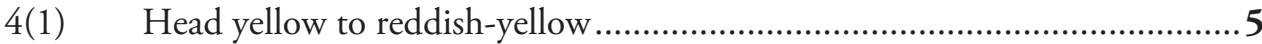

- Head dark brown to black ………...........................................................

5(4) Mesosoma with dark brown areas on anteromesoscutum and mesopleuron (Figs 54, 56, 58); dark brown coloration on posterior margin of mediotergite 3 and most of mediotergites 4-6 (Figs 54, 55, 57, 59) [Brazil, Paraguay] .....

Sendaphne paranaensis Scatolini \& Penteado-Dias, 1999

- Mesosoma uniformly orange-yellow to reddish-yellow; metasoma either entirely yellow or with brown bands on posterior margin of mediotergites $4-6$........... 6

6(5) Metasoma with brown bands on posterior margin of mediotergites 4-6 (Figs 50-53) [Brazil, French Guiana, Peru]....... Sendaphne olearus Nixon, 1965 Metasoma entirely yellow (Figs 31, 32, 35, 36) [Brazil, Ecuador, French Guiana] ............................................. Sendaphne jatai Penteado-Dias, 1995

7(4) Female metasoma either with extensive dark brown coloration on tergites 3+ or with some narrow brown bands on posterior margin of mediotergites 5-7

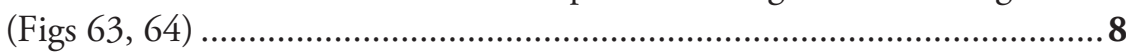

- $\quad$ Female metasoma entirely yellow (Figs 3, 4, 7, 18, 19, 22) ......................9

8(7) Metasoma with dark brown bands on posterior margin of mediotergites 3-6 and mediotergite 7 entirely dark brown to black; fore wing vein $1 \mathrm{Cu}-\mathrm{a}$ much shorter than vein $1 \mathrm{Cu}-\mathrm{b}$; T1 $10.0 \times$ as long as width at posterior margin; T1 $2.0 \times$ as long as T2; metacoxa $1.1 \times$ as long as metafemur.

Sendaphne brasilianus Penteado-Dias, 1995

Metasoma with some narrow brown bands on posterior margin of mediotergites 5-7 (Figs 63, 64); fore wing veins $1 \mathrm{Cu}-\mathrm{a}$ and $1 \mathrm{Cu}-\mathrm{b}$ subequal; $\mathrm{T} 1$ $6.0 \times$ as long as width at posterior margin; T1 $1.4 \times$ as long as T2; metacoxa $0.9-1.0 \times$ as long as metafemur

Sendaphne penteadodiasae Fernández-Triana \& Whitfield, sp. n.

9(7) Fore wing $\mathrm{r}$ and 2RS not clearly distinct from each other (Fig. 3); mediotergite 2 length $1.0 \times$ its width at posterior margin (Figs 3, 4, 7); face centrally orange (Fig. 2); ovipositor 1.8-1.9 $\times$ as long as metatibia [Ecuador]

Sendaphne anitae Fernández-Triana $\&$ Whitfield, sp. n.

- $\quad$ Fore wing with veins $r$ and 2RS clearly distinct from each other, and meeting at a sharp angle (not clearly visible in Fig. 17); mediotergite 2 length 1.8-2.0 
$x$ its width at posterior margin (Figs 18, 19); face entirely dark brown to black (Fig. 16); ovipositor 2.0-2.2 $\times$ as long as metatibia [Brazil, Colombia, or Mexico]

10(9) Distance between anatomical line tangent to posterior margin of anterior ocellus and anterior margin of posterior ocelli $0.5 \times$ diameter of anterior ocelli (Fig. 77); ocular-ocellar line $2.5 \times$ as long as posterior ocellus diameter; interocellar distance $1.6 \times$ as long as posterior ocellus diameter; T1 relatively narrower medially, T1 width at half length of tergite clearly less than width at anterior margin, and $1.5 \times$ as wide as width at posterior margin (Fig. 78); T2 $1.8 \times$ as long as wide [Mexico] Sendaphne sulmo Nixon, 1965 - $\quad$ Distance between anatomical line tangent to posterior margin of anterior ocellus and anterior margin of posterior ocelli $0.2-0.3 \times$ diameter of anterior ocelli (partially visible in Fig. 16); ocular-ocellar line 2.1-2.4 $\times$ as long as posterior ocellus diameter; interocellar distance $1.2-1.4 \times$ as long as posterior ocellus diameter; $\mathrm{T} 1$ relatively wider medially, $\mathrm{T} 1$ width at half length of tergite about same as width at anterior margin, and at least $2.0 \times$ as wide as width at posterior margin (Figs 18, 19); T2 $2.0 \times$ as long as wide [Brazil, Colombia]

Sendaphne dianariaspennae Fernández-Triana $\&$ Whitfield, sp. n.

\section{Taxonomic treatment of species, in alphabetical order}

Sendaphne anitae Fernández-Triana \& Whitfield, sp. n.

http://zoobank.org/5A29AD35-D955-44B1-B1C3-64182FD0E55B

Figs 1-7

Holotype. Female, CNC. ECUADOR, Napo, Reventador; 11.iii.1983; coll. L. Huggert. DNA Voucher code: CNCHYM 07027.

Paratypes. 4 o (CNC), same locality and collecting date than holotype. Two of the specimens also have DNA Voucher codes: CNCHYM 07026, CNCHYM 07028.

Diagnosis. This species is morphological similar to Sendaphne dianariaspennae and S. sulmo but differs from those species in the shape and angle of junction of veins $\mathrm{r}$ and 2RS, shape of mediotergite 2, ovipositor length, and head coloration (orangeyellow on clypeus and face centrally in anitae, head entirely dark brown to black in dianariaspennae and sulmo).

Description. Head color: dark brown to black, except for orange-yellow on clypeus and face centrally. Mesosoma color: orange-yellow. Tegula color: orange-yellow. Metasoma color (dorsally): yellow. Metacoxa color: yellow. Anatomical line tangent to posterior margin of anterior ocellus crossing very slightly (less than $0.01 \mathrm{~mm}$ ) above anterior margin of posterior ocelli. Ocular-ocellar line: $0.20 \mathrm{~mm}$. Interocellar distance: $0.09 \mathrm{~mm}$. Posterior ocellus diameter: $0.09 \mathrm{~mm}$. Body length: $4.0-4.5 \mathrm{~mm}$. Fore wing length: $3.6-4.1 \mathrm{~mm}$. Ovipositor length: $2.4-2.6 \mathrm{~mm}$. Metacoxa length: 1.3 $\mathrm{mm}$. Metafemur length: $1.2 \mathrm{~mm}$. Metatibia length: $1.3-1.4 \mathrm{~mm}$. T1 length/width at 


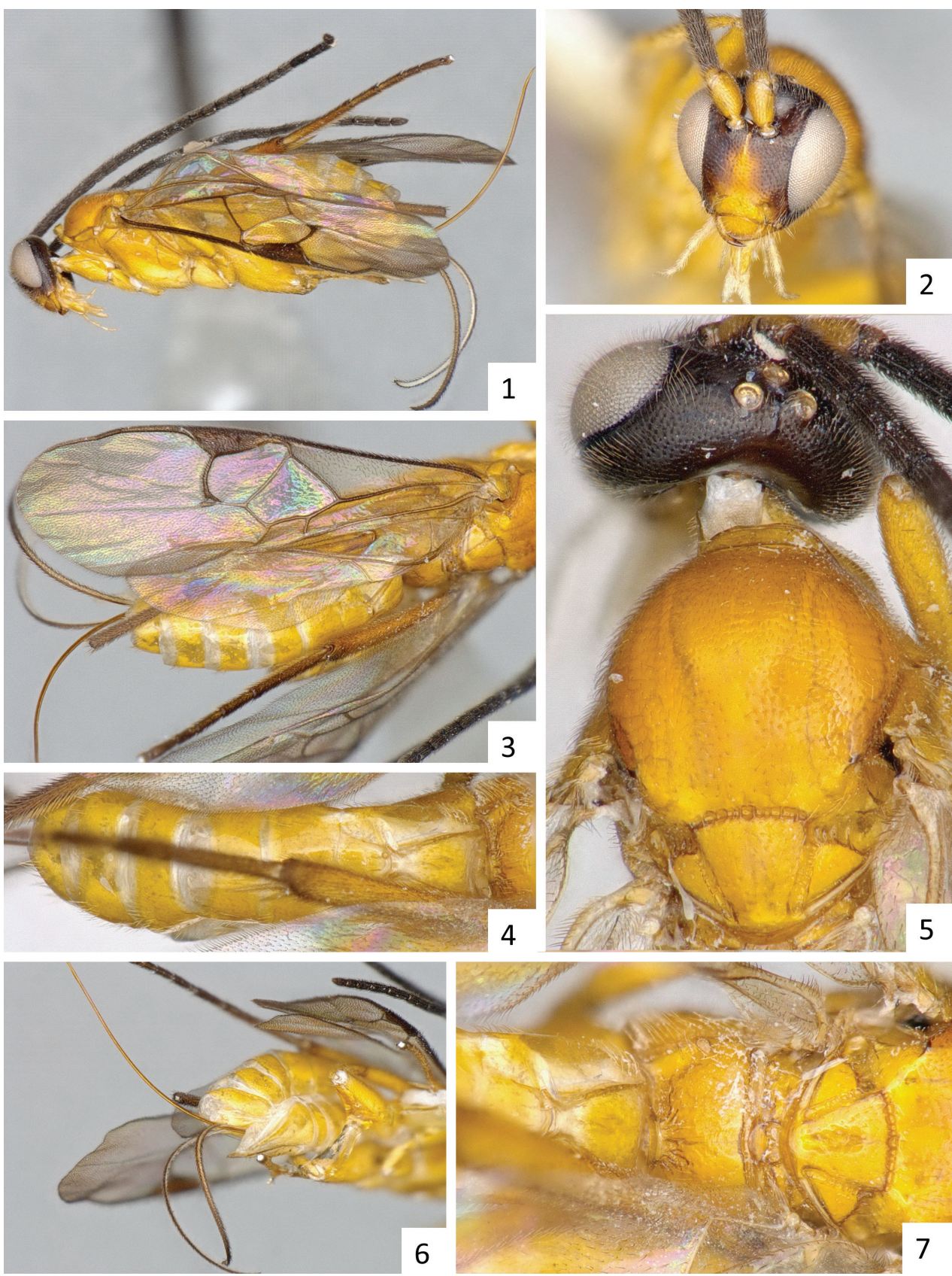

Figures I-7. Sendaphne anitae, female holotype. I Habitus, lateral view 2 Head, frontal view 3 Fore wing 4 Metasoma, dorsal view 5 Head and mesosoma (partially) lateral view $\mathbf{6}$ Metasoma, ventro-lateral view 7 Mesosoma and metasoma (partially) dorsal view. 
posterior margin: $0.6 \mathrm{~mm} / 0.15 \mathrm{~mm}$. T2 length/width at posterior margin: $0.26-0.30$ $\mathrm{mm} / 0.3 \mathrm{~mm}$.

Distribution. Only known from the type locality in Ecuador.

Molecular data. No DNA was recovered from the three specimens sampled.

Etymology. Named after Ana María (Anita) Fernández Galliano, daughter of the senior author, for being such a joyful and wonderful person.

Sendaphne bennetti Fernández-Triana \& Whitfield, sp. n.

http://zoobank.org/FB93B9D4-E93C-4939-9740-9C6901154A60

Figs $37-44$

Holotype. Male, CNC. MEXICO, Durango, 39km W of La Ciudad, 2120m; 2.vii.1964; coll. WRM Mason. DNA Voucher code: CNCHYM 07032.

Diagnosis. This species is morphologically similar to Sendaphne broadi from Ecuador, but $S$. bennetti has a wider mediotergite 1 (length $4.0 \times$ its width at posterior margin vs $6.0 \times$ in broadi), a less transverse first discal cell in the fore wing $(1.3 \times$ as wide as high vs $2.0 \times$ ), and a geographical distribution far apart.

Description. Head color: black. Mesosoma color: black. Tegula color: yellow. Metasoma color (dorsally): mediotergites 1, 2 and anterior half of 3 yellowish-red, rest dark brown to black. Metacoxa color: yellow. Anatomical line tangent to posterior margin of anterior ocellus crossing beneath anterior margin of posterior ocelli. Ocular-ocellar line: $0.21 \mathrm{~mm}$. Interocellar distance: $0.14 \mathrm{~mm}$. Posterior ocellus diameter: $0.09 \mathrm{~mm}$. Body length: $4.1 \mathrm{~mm}$. Fore wing length: $4.0 \mathrm{~mm}$. Metacoxa length: $1.1 \mathrm{~mm}$. Metafemur length: $1.2 \mathrm{~mm}$. Metatibia length: $1.4 \mathrm{~mm}$. T1 length/ width at posterior margin: $0.60 \mathrm{~mm} / 0.12 \mathrm{~mm}$. T2 length/width at posterior margin: $0.35 \mathrm{~mm} / 0.35 \mathrm{~mm}$.

Distribution. Only known from the type locality in Mexico.

Molecular data. No DNA could be recovered from the specimen sampled.

Comments. Even though only one male specimen is known, it is sufficiently distinctive to warrant description. This species is the northernmost known distribution of the genus Sendaphne.

Etymology. Named after Dr. Andrew Bennett of the Canadian National Collection, Ottawa, and Canadian expert on Ichneumonidae, in appreciation for his support and encouragement to study braconid wasps.

Sendaphne brasilianus Penteado-Dias, 1995: 251.

Holotype. Female, DCBU. BRAZIL. Distrito Federal, Brasília, xii-1981, Malaise trap (not examined). 

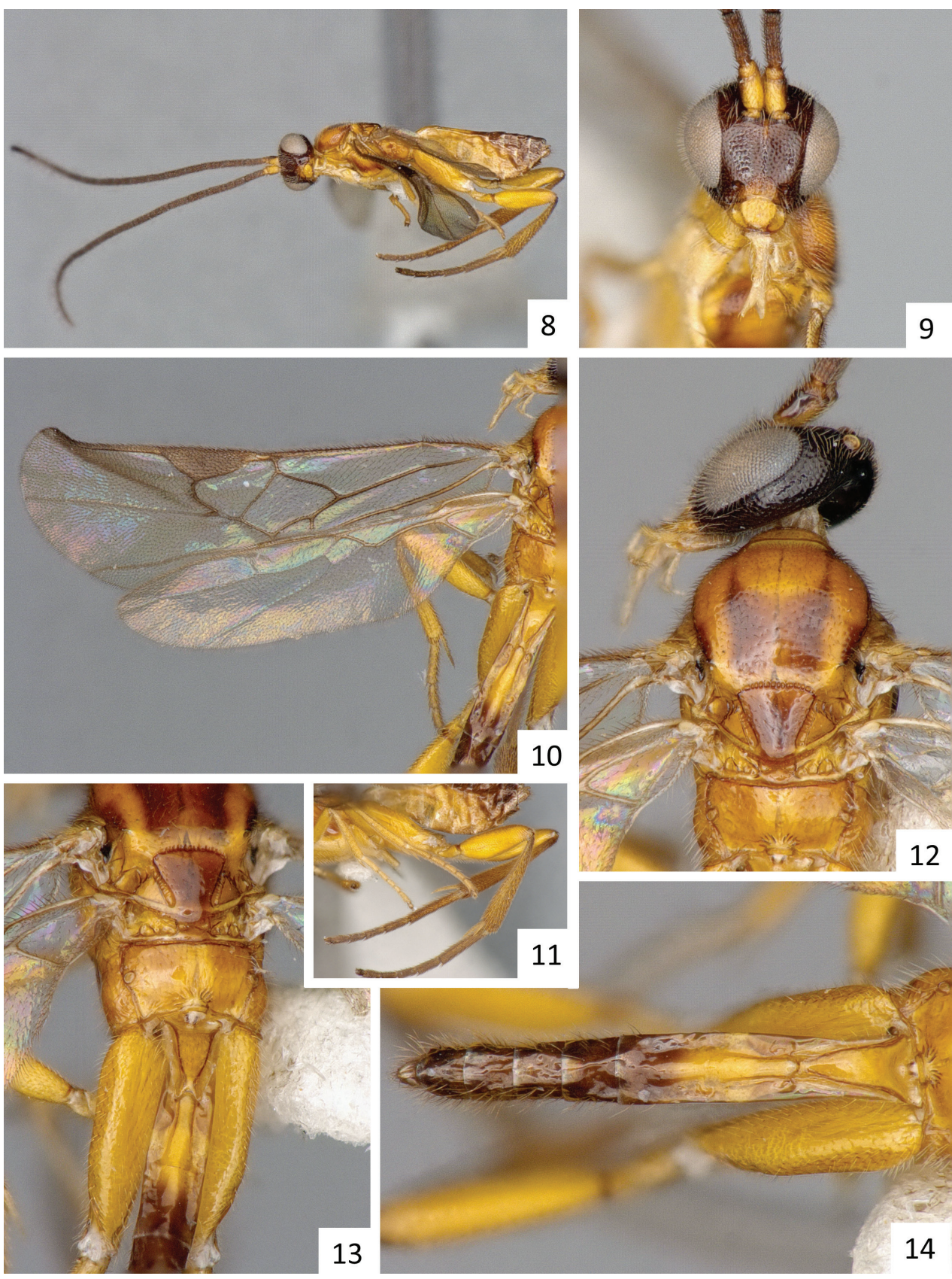

Figures 8-I4. Sendaphne sulmo, male specimen from Mexico. 8 Habitus, lateral view 9 Head, frontal view $\mathbf{I} \mathbf{0}$ Fore wing II Meso- and metasoma (partially), lateral view, and hind legs $\mathbf{2} \mathbf{2}$ Head and mesosoma, dorsal view 13 Meso- and metasoma (partially), dorsal view 14 Metasoma, dorsal view. 
Diagnosis. This species is morphologically similar to Sendaphne penteadodiasae but it has a slightly different color pattern, fore wing vein $1 \mathrm{Cu}-\mathrm{a}$ much shorter than vein $1 \mathrm{Cu}-\mathrm{b}$ (subequal in penteadodiasae), much longer and narrow $\mathrm{T} 1$, and slightly longer metacoxa.

Distribution. Only known from the type locality in Brazil.

Molecular data. No specimen is known to have been sampled for DNA.

Comments. We could not study a specimen of this species, but the original description is sufficiently detailed for recognition, including several line drawings of the metasoma, fore wing, tip of antenna and hind leg (Penteado-Dias 1995).

\section{Sendaphne broadi Fernández-Triana \& Whitfield, sp. n.} http://zoobank.org/B0C5F4FA-6171-4DFC-93B3-293898C2E94B Figs $23-28$

Holotype. Male, CNC. ECUADOR, Napo, $5 \mathrm{~km} \mathrm{~S} \mathrm{of} \mathrm{Baeza,} \mathrm{1,700} \mathrm{m;} \mathrm{13.ii.1983;}$ coll. Masner \& Sharkey. DNA Voucher code: CNCH3323.

Paratypes. 2 ( $\mathrm{CNC})$. One specimen with same locality and collecting date than holotype, the other collected at $1900 \mathrm{~m}$ on 9.ii.1983. DNA Voucher codes: $\mathrm{CNCH} 3322$ and $\mathrm{CNCH} 3324$.

Diagnosis. This species is morphologically similar to Sendaphne bennetti from Mexico, but $S$. broadi has a narrower mediotergite 1 (length $6.0 \times$ its width at posterior margin vs $4.0 \times$ in bennetti), a more transverse first discal cell in the fore wing (2.0 $x$ as wide as high vs $1.3 \times$ ), and a geographical distribution far apart.

Description. Head color: dark brown. Mesosoma color: dark brown. Tegula color: yellow. Metasoma color (dorsally): mediotergites 1-2 yellowish-brown, rest brown to dark brown. Metacoxa color: yellow. Anatomical line tangent to posterior margin of anterior ocellus crossing very slightly (less than $0.01 \mathrm{~mm}$ ) above anterior margin of posterior ocelli. Ocular-ocellar line: $0.17 \mathrm{~mm}$. Interocellar distance: $0.09 \mathrm{~mm}$. Posterior ocellus diameter: $0.07 \mathrm{~mm}$. Body length: $3.5-3.7 \mathrm{~mm}$. Fore wing length: 3.3-3.5 $\mathrm{mm}$. Metacoxa length: $1.1 \mathrm{~mm}$. Metafemur length: $1.2 \mathrm{~mm}$. Metatibia length: 1.4 $\mathrm{mm}$. T1 length/width at posterior margin: $0.45 \mathrm{~mm} / 0.06-0.07 \mathrm{~mm}$. T2 length/width at posterior margin: $0.25 \mathrm{~mm} / 0.20 \mathrm{~mm}$.

Distribution. Ecuador.

Molecular data. No DNA could be recovered from the three specimens sampled.

Comments. Even though only male specimens of this species are known, they are sufficiently distinctive to warrant description.

Etymology. Named after Dr. Gavin Broad, of the Natural History Museum, London, England, and British expert on Ichneumonidae, in appreciation for his support over the years, including sharing pictures of and facilitating access to type material deposited in London. 

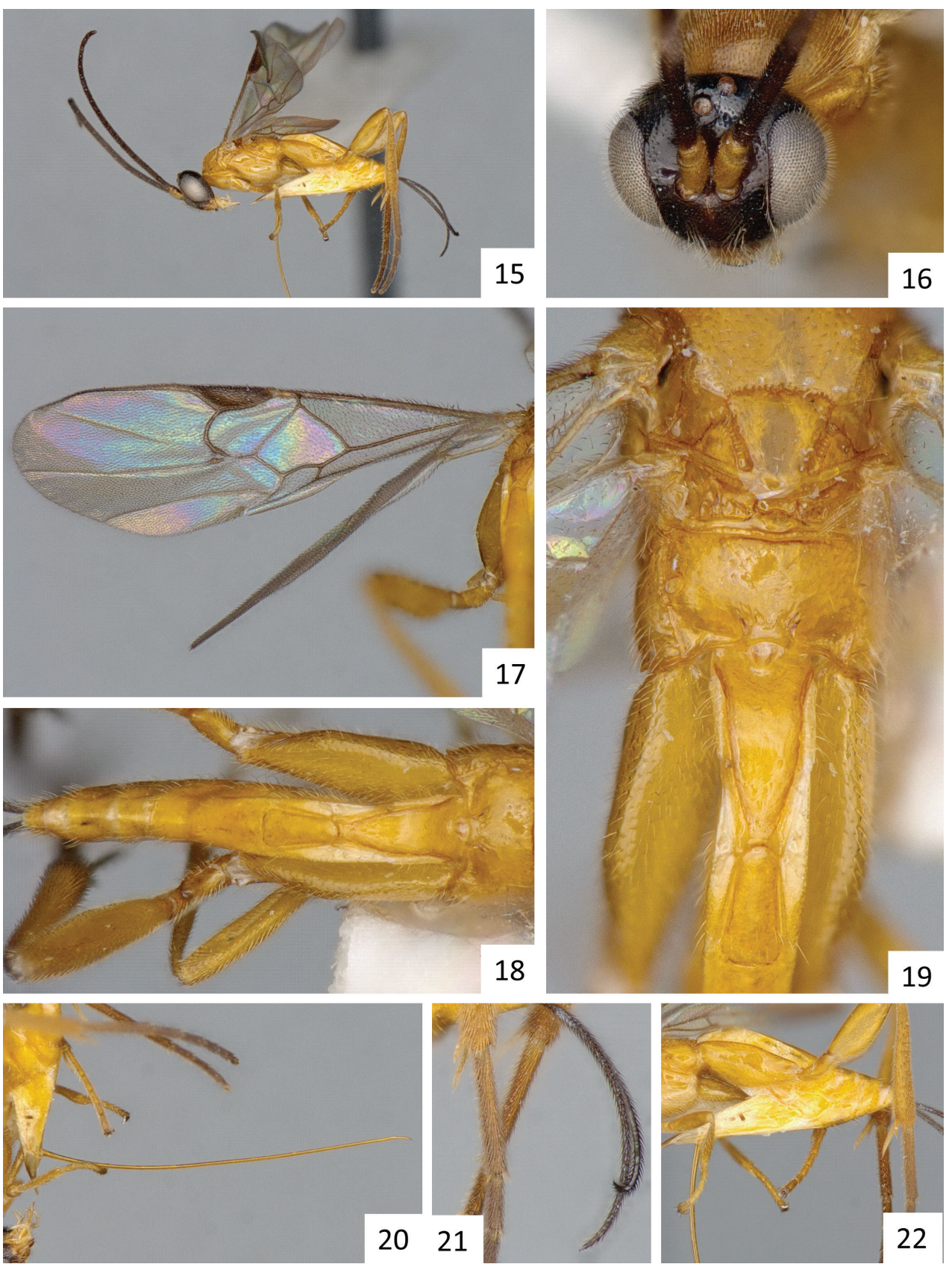

Figures I5-22. Sendaphne dianariaspennae, female holotype. 15 Habitus, lateral view 16 Head, frontal view (some inclination downwards) 17 Fore wing 18 Propodeum and metasoma, dorsal view 19 Meso- and metasoma (partially), dorsal view. 20-22: Details of the ovipositor, ovipositor sheaths, and hypopygium. 


\section{Sendaphne dianariaspennae Fernández-Triana \& Whitfield, sp. n.} http://zoobank.org/423FC0AA-4087-414C-B242-856191CFF5AD

Figs 15-22

Holotype. Female, CNC. BRAZIL, Rio de Janeiro, Mangaratiba; i.1976; coll. M. Alvarenga. DNA Voucher code: CNCHYM 07025.

Paratypes. 3 q, $1 \hat{\jmath}$ (CNC), Brazil, same locality and date than holotype. 1 ? (CNC), Brazil, Nova Teutonia, 27ำ1'S, 52²3'W, 300-500m, 21.iii.1961, coll. F. Plaumann. 2 đo (CNC), Brazil, Guanabara and Represa Rio Grande, i.1969 and

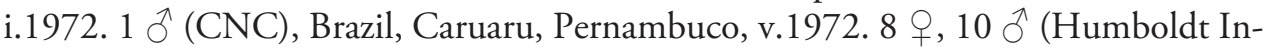
stitute), Colombia, Magdalena, PNN Tayrona Zaino, $50 \mathrm{~m}, 11^{\circ} 20^{\prime} \mathrm{N}, 74^{\circ} 2^{\prime} \mathrm{W}$, specimens collected between 13.v.2000 and 30.viii.2000, coll. R. Henriquez.

Diagnosis. This species is morphological similar to Sendaphne sulmo but differs in the smaller, less separated ocelli (which form a lower triangle, compared to higher triangle in sulmo), T1 relatively wider medially, and T2 relatively slender than sulmo. The geographical distribution of the two species is more than 2,000 km apart.

Description. Head color: dark brown to black. Mesosoma color: orange-yellow. Tegula color: orange-yellow. Metasoma color (dorsally): yellow. Metacoxa color: yellow. Anatomical line tangent to posterior margin of anterior ocellus crossing slightly $(0.01-0.02 \mathrm{~mm})$ above anterior margin of posterior ocelli. Ocular-ocellar line: 0.17 $\mathrm{mm}$. Interocellar distance: $0.10 \mathrm{~mm}$. Posterior ocellus diameter: 0.07-0.08 mm. Body length: 3.2-3.5 mm. Fore wing length: 3.0-3.2 mm. Ovipositor length: 2.3-3.0 mm. Metacoxa length: 0.90-0.95 mm. Metafemur length: $0.90 \mathrm{~mm}$. Metatibia length: $1.12-1.35 \mathrm{~mm}$. T1 length/width at posterior margin: $0.45-0.50 \mathrm{~mm} / 0.05-0.06 \mathrm{~mm}$. T2 length/width at posterior margin: $0.35 \mathrm{~mm} / 0.18-0.20 \mathrm{~mm}$.

Distribution. Brazil and Colombia.

Molecular data. Four of the paratypes from Brazil (DNA Voucher codes: CNCHYM 07023, CNCHYM 07024 and CNCHYM 07040) as well as the holotype were sampled for DNA. Only one of the paratypes (CNCHYM 07040) rendered a minibarcode of 103 base pairs.

Comments. The Brazilian specimens of $S$. dianariaspennae were collected between January and March, while the Colombian specimens were collected between May and August.

Etymology. Named after Diana Carolina Arias-Penna (Colombia), in recognition of her blossoming career studying neotropical Braconidae, especially species from South America.

\section{Sendaphne jatai Penteado-Dias, 1995: 252.}

Figs 29-36

Holotype. Female, DCBU. BRAZIL. Sao Paulo, Reserva Ecológica do Jataí, gallery forest of Mogi River; 24.v.1991; sweeping (not examined). 

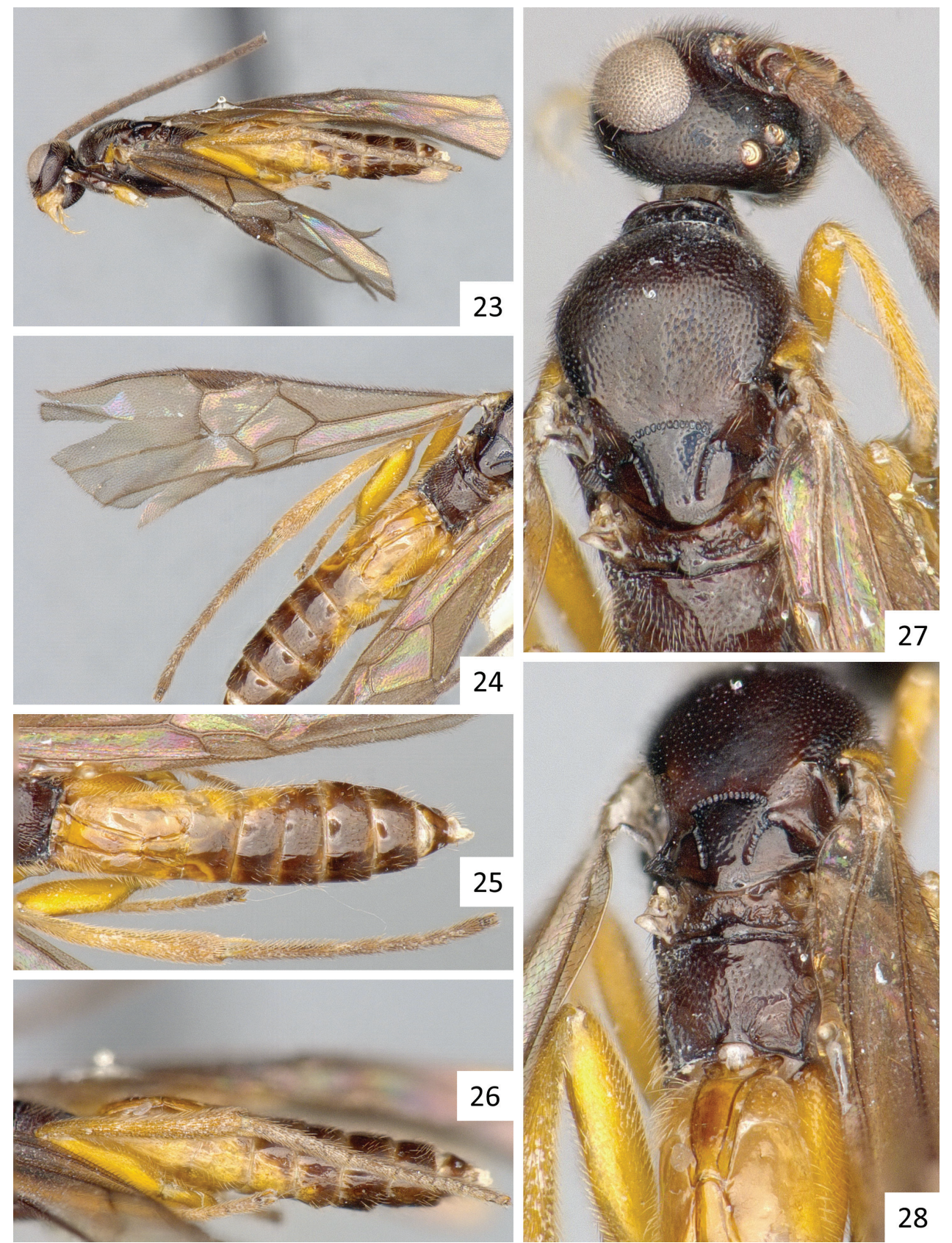

Figures 23-28. Sendaphne broadi, male holotype. 23 Habitus, lateral view 24 Fore wing $\mathbf{2 5}$ Metasoma, dorsal view $\mathbf{2 6}$ Metasoma, lateral view $\mathbf{2 7}$ Head and mesosoma (partially), dorsal view $\mathbf{2 8}$ Mesosoma and T1-T2, dorsal view. 

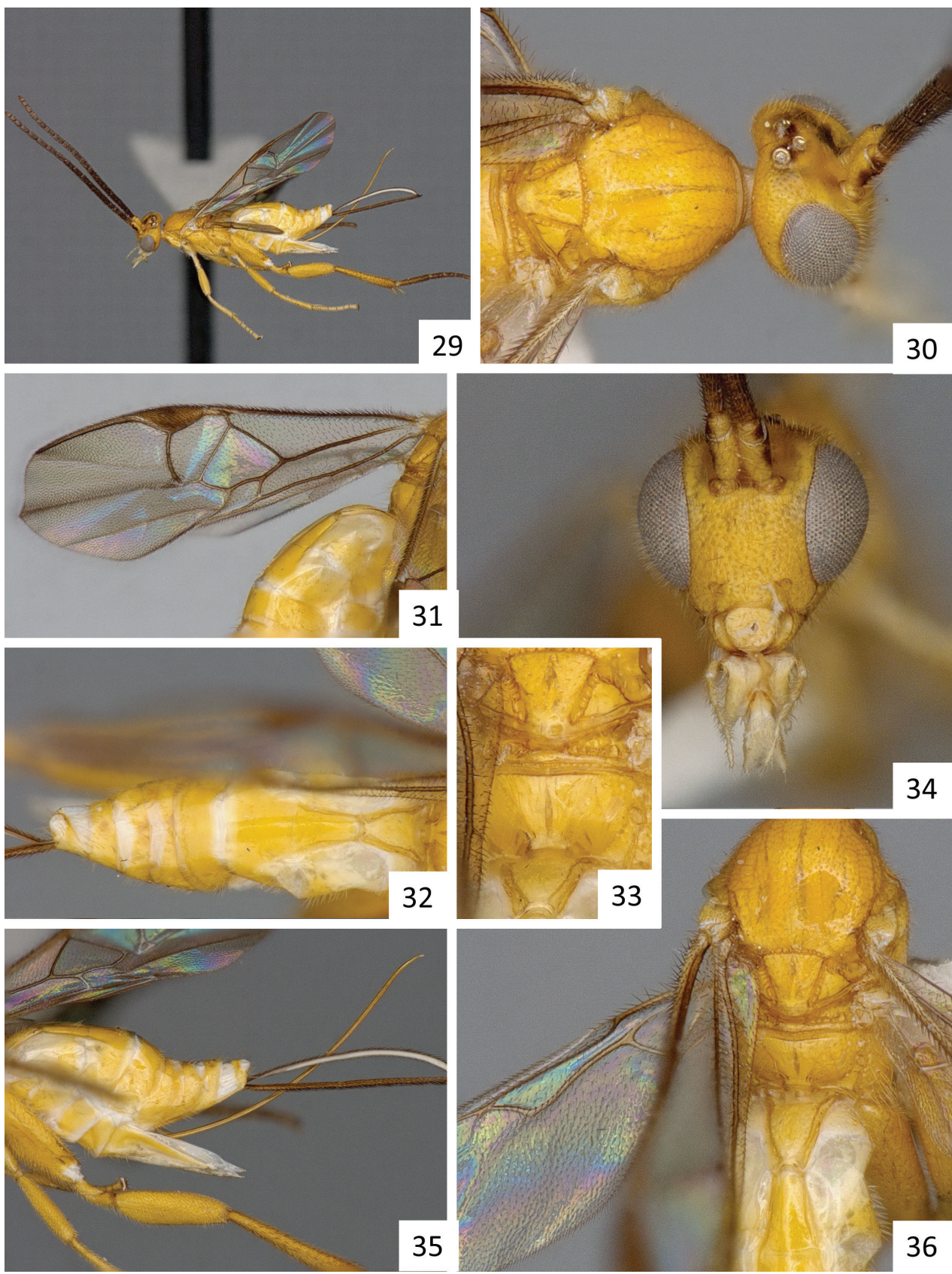

Figures 29-36. Sendaphne jatai, female specimen from Brazil. 29 Habitus, lateral view $\mathbf{3 0}$ Head and mesosoma (partially), dorso-lateral view 3I Fore wing 32 Metasoma, dorsal view 33 Scutellar disc, propodeum and T1 (partially), dorsal view 34 Head, frontal view 35 Metasoma, lateral view 36 Mesosoma and metasoma (partially), dorsal view. 

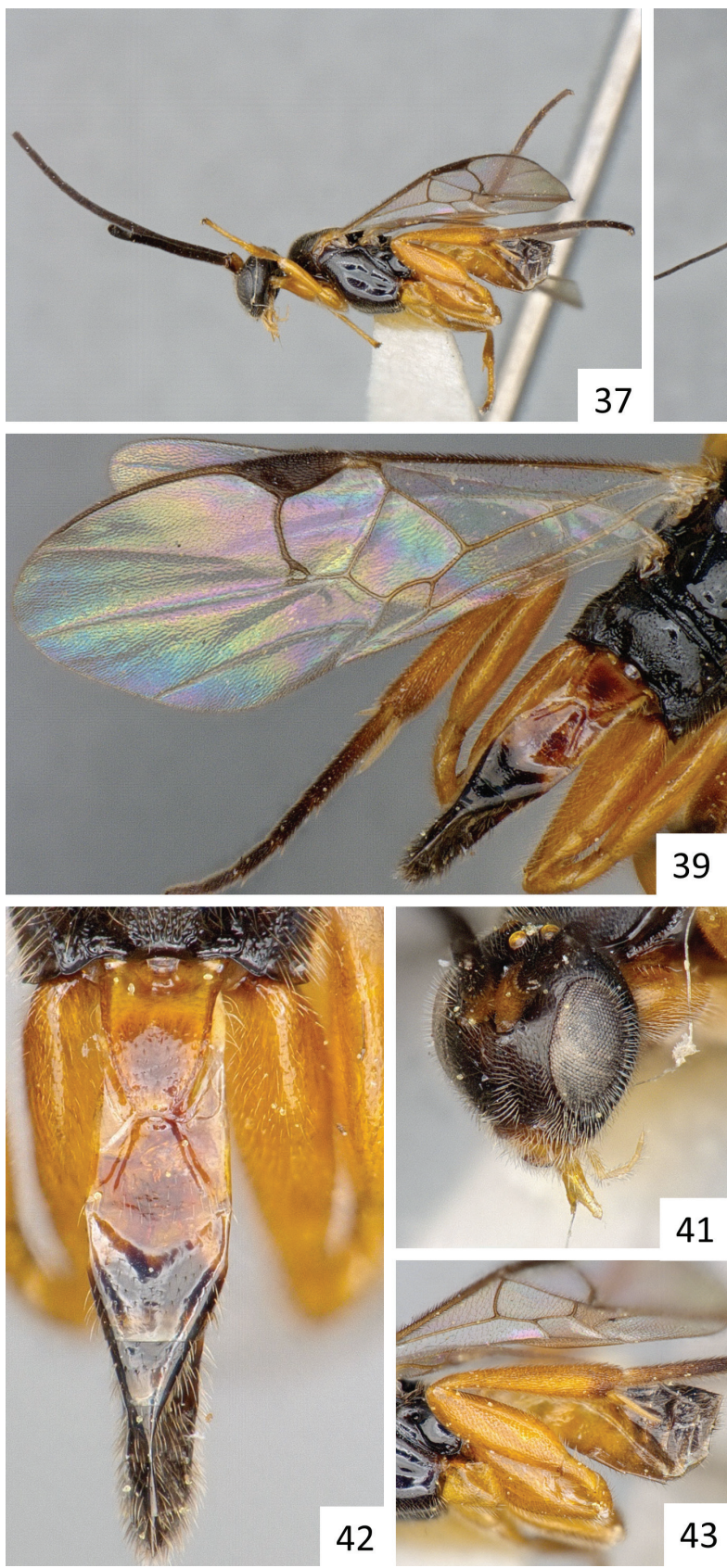

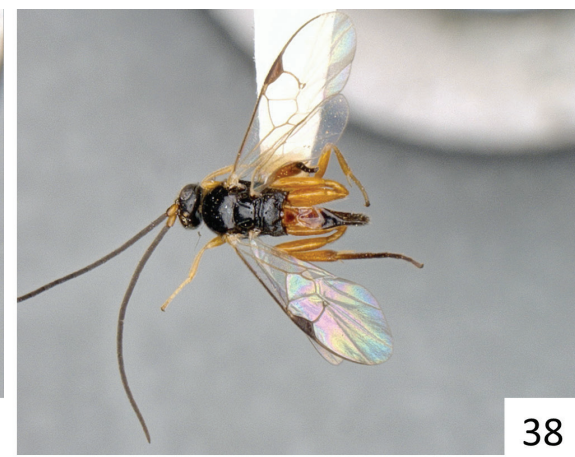

38
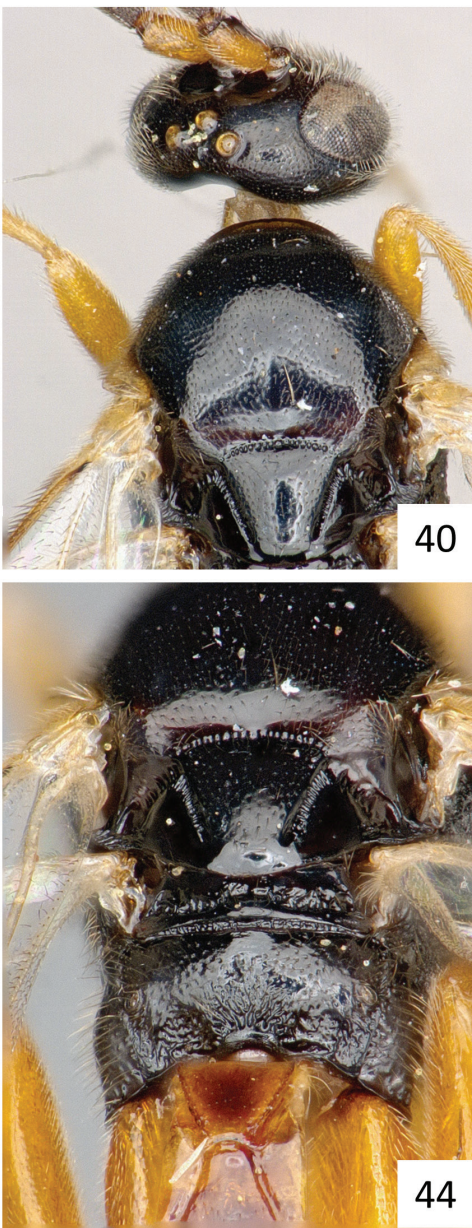

Figures 37-44. Sendaphne bennetti, male holotype. 37 Habitus, lateral view 38 Habitus, dorsal view 39 Fore wing $\mathbf{4 0} \mathrm{Head}$ and mesosoma (partially), dorsal view $\mathbf{4 I}$ Head, fronto-lateral view $\mathbf{4 2}$ Metasoma, dorsal view $\mathbf{4 3}$ Metasoma, lateral view $\mathbf{4 4}$ Scutellar disc, propodeum and T1-T2 (partially), dorsal view. 
Specimens examined. 1 (CNC), Brazil, Mato Grosso, Sinop, x.1974, coll. M. Alvarenga, DNA Voucher code: $\mathrm{CNCH} 3320.1$ q, 1 ते (CNC), Ecuador, Pichincha, $47 \mathrm{~km}$ S of Santo Domingo, Rio Palenque, 200m, 18-30.v.1975 and 22-31.vii.1976, coll. S. \& J. Peck, DNA Voucher codes: CNCHYM 07034 and CNCHYM 07035. 1 ㅇ (FUSAGx), French Guiana, Saul, Crique popote, Mont Belvédere, $3^{\circ} 36^{\prime} \mathrm{N}$, $53^{\circ} 10^{\prime} \mathrm{W}$, ii.2001, coll. J. Tarin.

Diagnosis. This is the only species where female specimens have the body entirely yellow.

Distribution. Brazil, Ecuador, French Guiana.

Molecular data. The two specimens from Ecuador rendered minibarcodes of 102 base pairs (CNCHYM 07034) and 164 base pairs (CNCHYM 07035).

Comments. The male specimen from Ecuador has some dark bands on mediotergites $5-7$, but otherwise is similar to the original description.

\section{Sendaphne olearus Nixon, 1965: 204.}

Figs $45-53,79-80$

Holotype. Female, BMNH. BRAZIL, Nova Teutonia, 2.iii.1937 (not examined).

Specimens examined. 1 \& (CNC), Brazil, Nova Teutonia, $27^{\circ} 11^{\prime} \mathrm{S}, 52^{\circ} 23^{\prime} \mathrm{W}$, 300-500m, 2.iv.1966, DNA Voucher code: CNCHYM 07019. 3 ○ (FUSAGx), French Guiana, Kaw Mountain, Patawa, 4³2'42.20"N, 5209'09.19"W, v.1999 and viii.1999, Malaise trap. 1 \& (BMNH) Peru, Loreto, Estacion Jenaro Herrera, 453'55.0"S, 73³9'00.4"W, 121m, 13-23.i.2011, D. Karlsson \& N. Dale-Skey, BMNH(E) 2011-72, BMNH(E)\#1249978.

Diagnosis. This species is similar to Sendaphne jatai, both having the lightest (i.e., more yellowish) coloration among all known species within the genus. S. olearus has brown bands on posterior margin of mediotergites $4-6$, and its body coloration is generally more yellow-reddish (jatai body color is entirely yellow).

Distribution. Brazil, French Guiana, Peru.

Molecular data. The CNC specimen from the type locality in Brazil (CNCHYM 07019) rendered a minibarcode of 164 base pairs.

Comments. We could not see the holotype of this species, but the original description is adequately detailed for discrimination. We examined one female from the type locality (collected 30 years after the holotype female), the only known male specimens (from French Guiana), which were discussed in Braet (2006), and the first known specimen from Peru.

\section{Sendaphne paranaensis Scatolini \& Penteado-Dias, 1999: 53.}

Figs 54-60

Holotype. Female, UFPR. BRAZIL, Reserva Biologica Samuel Klabin, Malaise trap (not examined). 

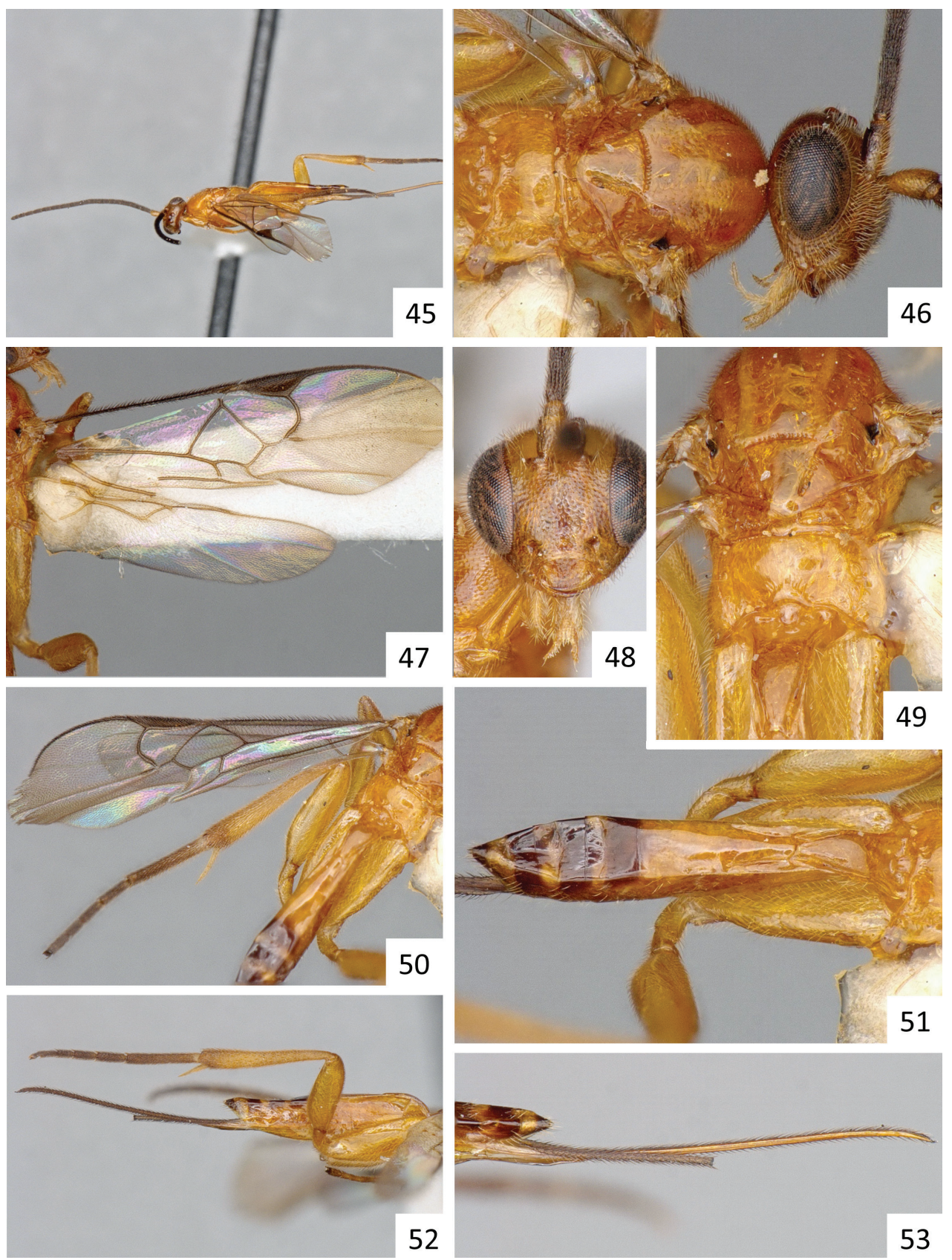

Figures 45-53. Sendaphne olearus, female specimen from Brazil. 45 Habitus, lateral view 46 Head and mesosoma, lateral view 47 Fore wing $48 \mathrm{Head}$, frontal view 49 Mesosoma and T1, dorsal view $\mathbf{5 0}$ Mesosoma and metasoma (partially), dorsal view $\mathbf{5}$ I Metasoma, dorsal view $\mathbf{5 2}$ Metasoma, lateral view 53 Details of the ovipositor and ovipositor sheaths. 

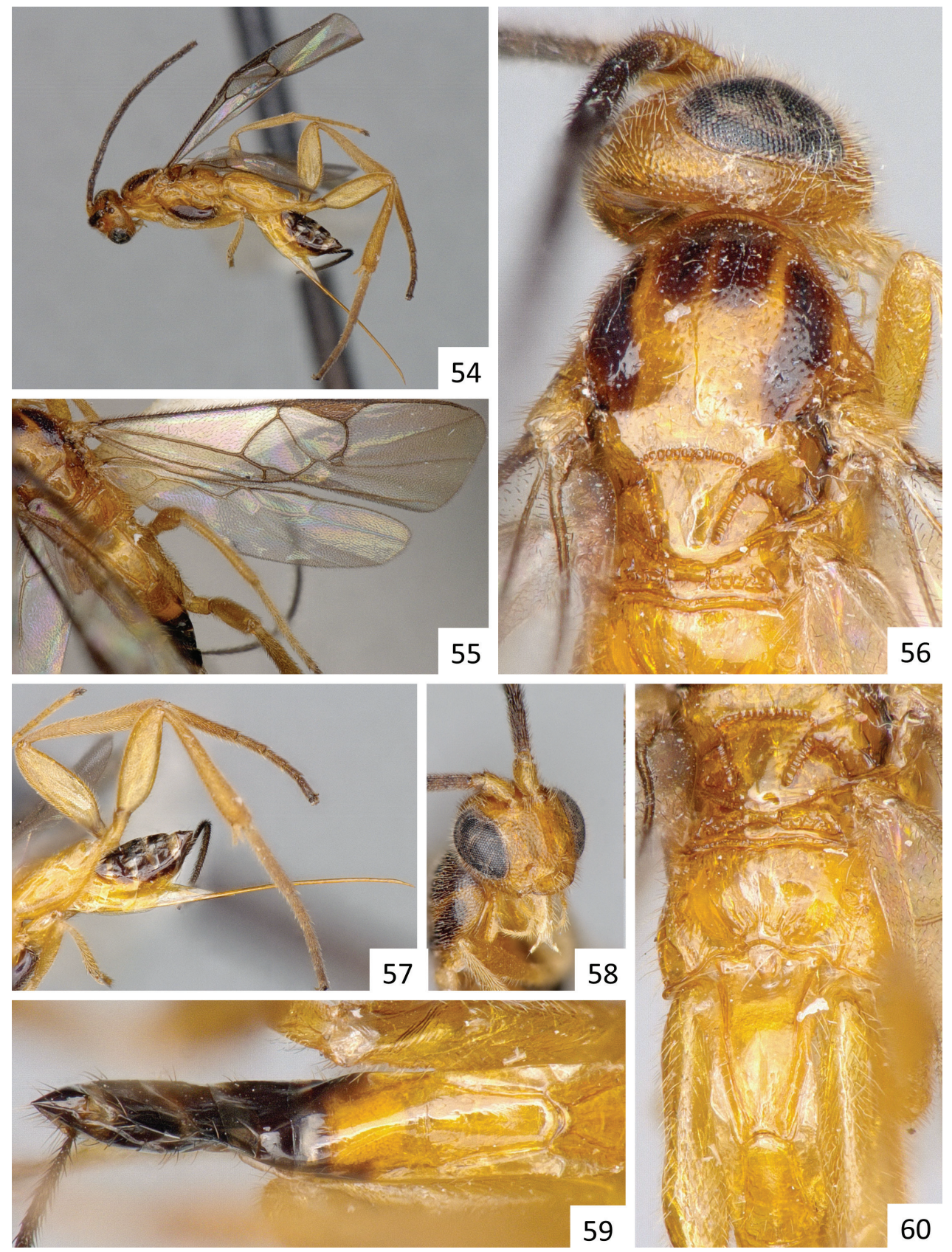

Figures 54-60. Sendaphne paranaensis, female specimen from Brazil. 54 Habitus, lateral view 55 Fore wing 56 Head and mesosoma (partially), dorsal view 57 Metasoma and legs, lateral view 58 Head, frontal view 59 Metasoma (partially), dorsal view 60 Scutellar disc, propodeum and T1-T2 (partially), dorsal view. 
Specimens examined. 1 ( $\mathrm{CNC}$, Brazil, Rio de Janeiro, Guanabara, Silva Jardim, iii.1974, DNA Voucher code: CNCHYM 07022. 1 đ (CNC), Brazil, Espirito Santo, Castello, xi.1976, DNA Voucher code: CNCHYM 07039. 2 ô (CNC), Paraguay, Pirapo, 1-3.i.1972, DNA Voucher codes: CNCHYM 07037, and CNCHYM 07038.

Other material mentioned in the original description. Almost 70 specimens (females and males) from Brazil, Paraná, Telêmaco Borba, collecting dates between viii.1986 and iii.1987.

Diagnosis. S. paranaensis is one of only three Sendaphne species with head entirely yellow (or yellow-orange). It differs from the other two species (S. jatai and $S$. olearus) in having dark brown areas on the anteromesoscutum and mesopleuron, and mediotergites 4+ entirely black.

Distribution. Brazil, Paraguay.

Molecular data. Of the four specimens in the CNC sampled for DNA, only one (CNCHYM 07037) rendered a minibarcode of 164 base pairs.

Comments. The male specimens from Paraguay are much darker in coloration, but similar variation is mentioned in the original description for the male paratypes from Brazil (Scatolini and Penteado-Dias 1999: 53).

\section{Sendaphne penteadodiasae Fernández-Triana \& Whitfield, sp. n.} http://zoobank.org/7FDD9815-D39B-49EE-9645-749C02277F24 Figs 61-67

Holotype. Female, CNC. BRAZIL, Campina Grande, near Curitiba, 15.ii.1966, coll. H. \& M. Townes. DNA Voucher code: CNCHYM 07020.

Paratype. $1 \hat{\delta}$, same locality as holotype, 10.ii.1966. DNA Voucher code: CNCHYM 07021.

Diagnosis. This species is morphologically similar to Sendaphne brasilianus but it has a slightly different color pattern, fore wing vein $1 \mathrm{Cu}-\mathrm{a}$ subequal to vein $1 \mathrm{Cu}-\mathrm{b}$ (much shorter in brasilianus), shorter and wider T1, and slightly shorter metacoxa.

Description. Head color: dark brown to black. Mesosoma color: orange-yellow. Tegula color: orange-yellow. Metasoma color (dorsally): mostly orange-yellow, with some narrow brown bands on posterior margin of mediotergites 5-7. Metacoxa color: yellow. Anatomical line tangent to posterior margin of anterior ocellus crossing very slightly (less than $0.01 \mathrm{~mm}$ ) above anterior margin of posterior ocelli. Ocular-ocellar line: $0.12 \mathrm{~mm}$. Interocellar distance: $0.10 \mathrm{~mm}$. Posterior ocellus diameter: 0.08 $\mathrm{mm}$. Body length: $4.2 \mathrm{~mm}$. Fore wing length: $3.9 \mathrm{~mm}$. Ovipositor length: $2.5 \mathrm{~mm}$. Metacoxa length: $1.20 \mathrm{~mm}$. Metafemur length: $1.25 \mathrm{~mm}$. Metatibia length: $1.11 \mathrm{~mm}$. T1 length/width at posterior margin: $0.35 \mathrm{~mm} / 0.06 \mathrm{~mm}$. T2 length/width at posterior margin: $0.25 \mathrm{~mm} / 0.12 \mathrm{~mm}$.

Distribution. Only known from the type locality in Brazil.

Molecular data. No DNA could be recovered from the two specimens sampled. 
Comments. The specimens of this species (housed in the CNC) were previously identified by W.R.M. Mason as "Sendaphne sulmo". However, the morphological differences from the original description of Sendaphne sulmo (see key and diagnosis above), and the disparate geographical distribution allows us to consider the Brazilian and Mexican specimens as separate species.

Etymology. Named after Dr. Angélica Maria Penteado Martins-Dias (Brazil), in recognition of her career studying Braconidae, and also for her work describing most of the previously known species of Sendaphne.

\section{Sendaphne rogerblancoi Fernández-Triana $\&$ Whitfield, sp. $\mathbf{n}$.} http://zoobank.org/09ABE6F1-4EFC-43B5-BE10-5631E671B76E Figs 68-75

Holotype. Female, CNC. COSTA RICA, Guanacaste, Area de Conservación Guanacaste, Sector Cacao, Sendero Cima, 1,460 m, 10.93328, -85.45729; 18.xii.2008; coll. D.H. Janzen \& W. Hallwachs. DNA Voucher code: DHJPAR0031465.

Paratypes. 4 ㅇ, 5 ㅇ (BMNH, CNC, INBio, NMNH), same locality as holotype. Collecting dates are mostly on December 2008, with one record each in November 2008, March 2009, and April 2009. All specimens are from one Malaise trap at the very peak of Volcán Cacao.

Diagnosis. This is the most distinctive species of Sendaphne based on coloration (head, mesosoma, metasoma, and metacoxa black), shape of first discal cell, and narrow mediotergite 1 .

Description. Head color: black. Mesosoma color: black. Tegula color: dark brown. Metasoma color (dorsally): black. Metacoxa color: dark brown to black. Anatomical line tangent to posterior margin of anterior ocellus crossing very slightly (less than $0.01 \mathrm{~mm}$ ) above anterior margin of posterior ocelli. Ocular-ocellar line: 0.17-0.18 mm. Interocellar distance: $0.09 \mathrm{~mm}$. Posterior ocellus diameter: $0.08 \mathrm{~mm}$. Body length: 4.3-4.6 mm. Fore wing length: 4.2-4.3 mm. Ovipositor length: 3.8-4.2 $\mathrm{mm}$. Metacoxa length: $1.10-1.11 \mathrm{~mm}$. Metafemur length: $0.85-0.90 \mathrm{~mm}$. Metatibia length: $1.5-1.6 \mathrm{~mm}$. T1 length/width at posterior margin: $0.80-0.85 \mathrm{~mm} / 0.15-0.16$ $\mathrm{mm}$. T2 length/width at posterior margin: $0.45 \mathrm{~mm} / 0.25 \mathrm{~mm}$.

Distribution. Only the summit cloud forest at $1,450 \mathrm{~m}$ on Volcán Cacao, northwestern Costa Rica.

Molecular data. In BOLD there are data for 16 specimens of this species (the holotype, the paratypes and other specimens that we could not examine) which rendered partial barcodes, most of them from 260 to 390 base pairs. Only the holotype (DNA Voucher code: DHJPAR0031465) had a longer barcode (633 base pairs).

Etymology. This unique species, the only Sendaphne known from Costa Rica so far, is named after Sr. Roger Blanco Segura, of Area de Conservación Guanacaste (ACG), northwestern Costa Rica, in recognition of his $3+$ decades of intense care and management of ACG in an enormous variety of circumstances and for a very large array of purposes. 

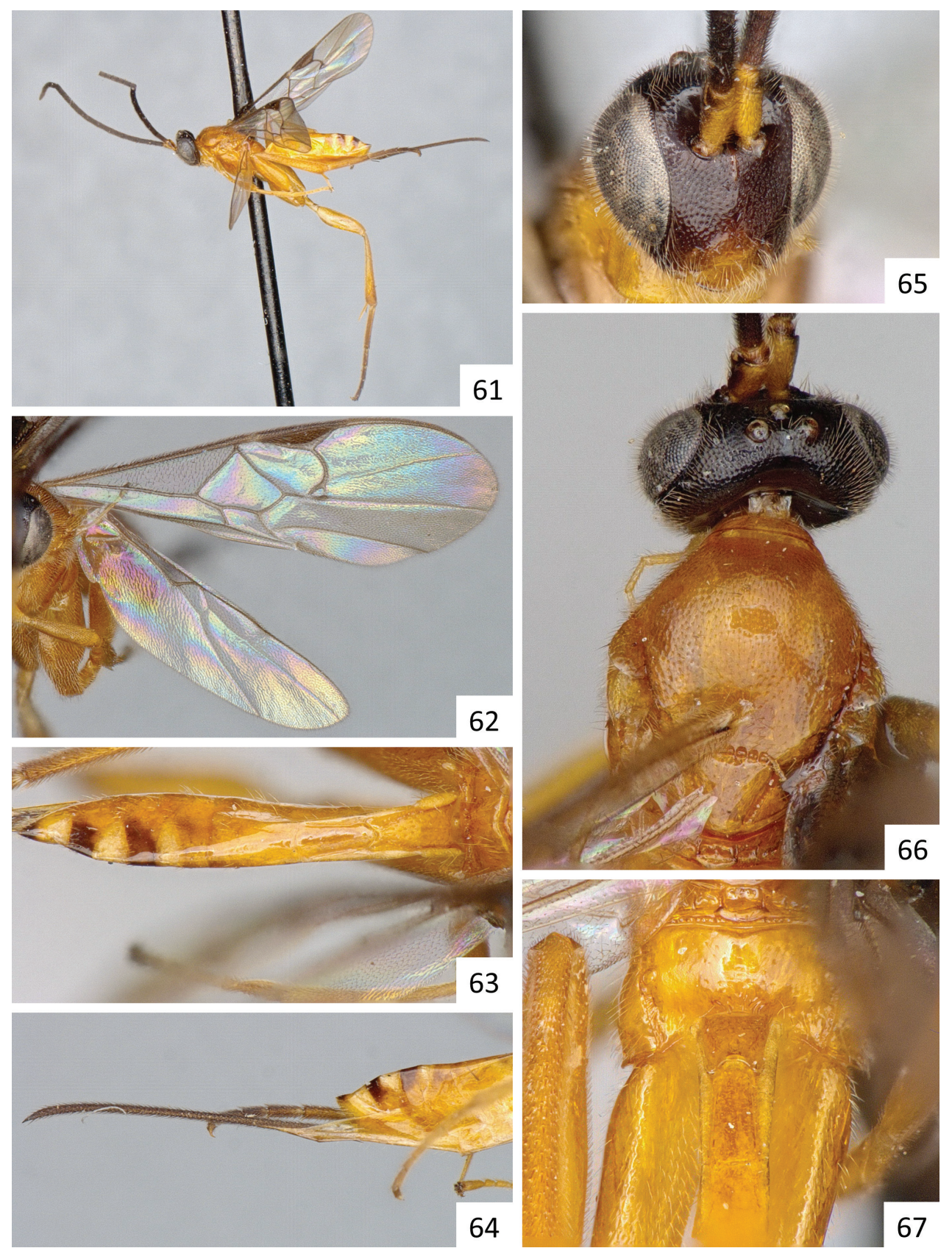

Figures 6I-67. Sendaphne penteadodiasae, female holotype. 6I Habitus, lateral view 62 Fore wing 63 Metasoma, dorsal view 64 Ovipositor and hypopygium, lateral view 65 Head, frontal view 66 Head and mesosoma (partially), dorsal view $\mathbf{6 7}$ Propodeum, T1-T2 (partially), dorsal view. 

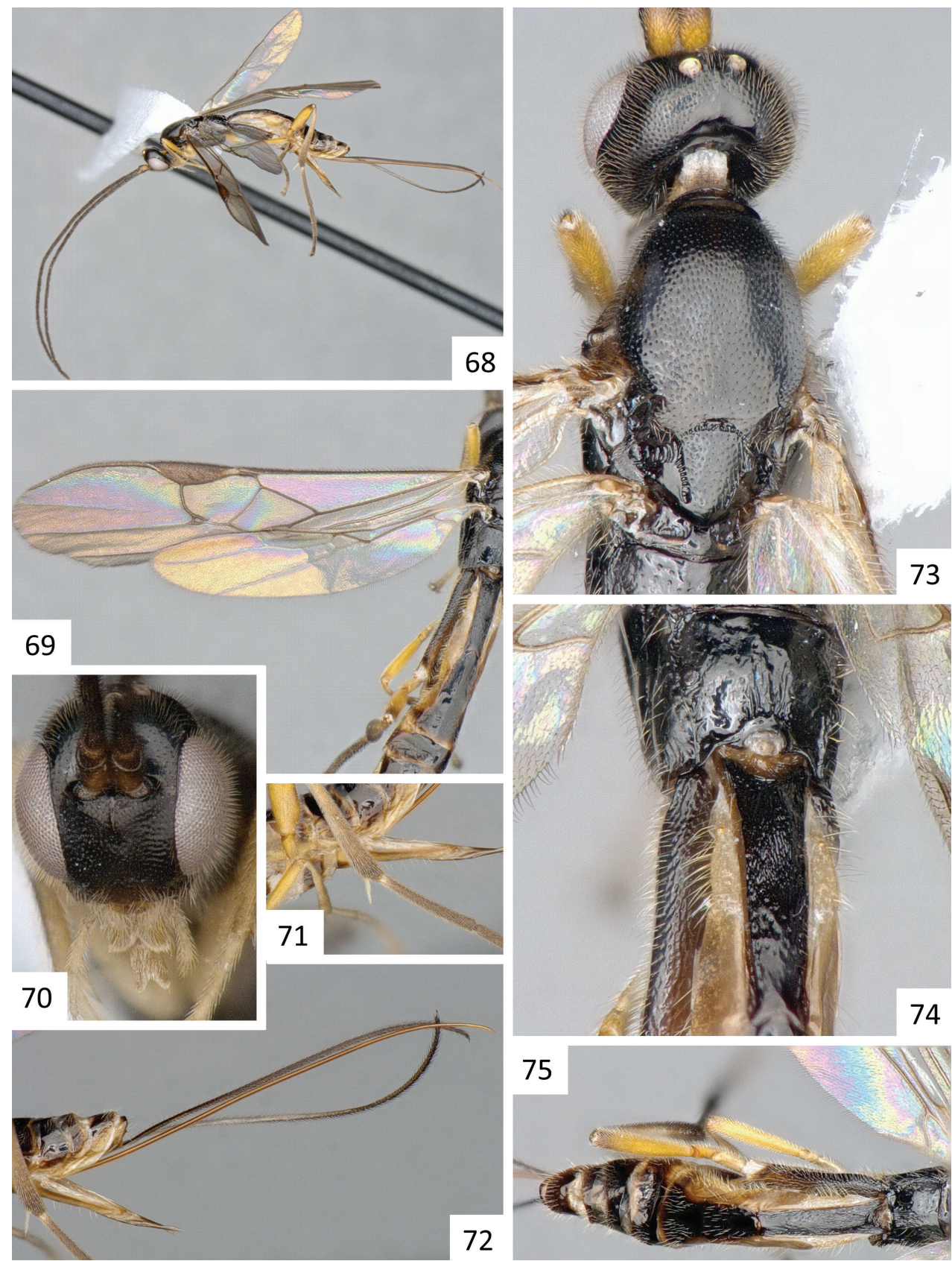

Figures 68-75. Sendaphne rogerblancoi, female holotype. 68 Habitus, lateral view 69 Fore wing 70 Head, frontal view 71 Hypopygium and hind legs (partially), lateral view 72 Ovipositor, ovipositor sheaths, and hypopygium, lateral view $\mathbf{7 3} \mathrm{Head}$ and mesosoma (partially), lateral view $\mathbf{7 4}$ Propodeum and T1, dorsal view 75 Propodeum and mesosoma, dorsal view. 


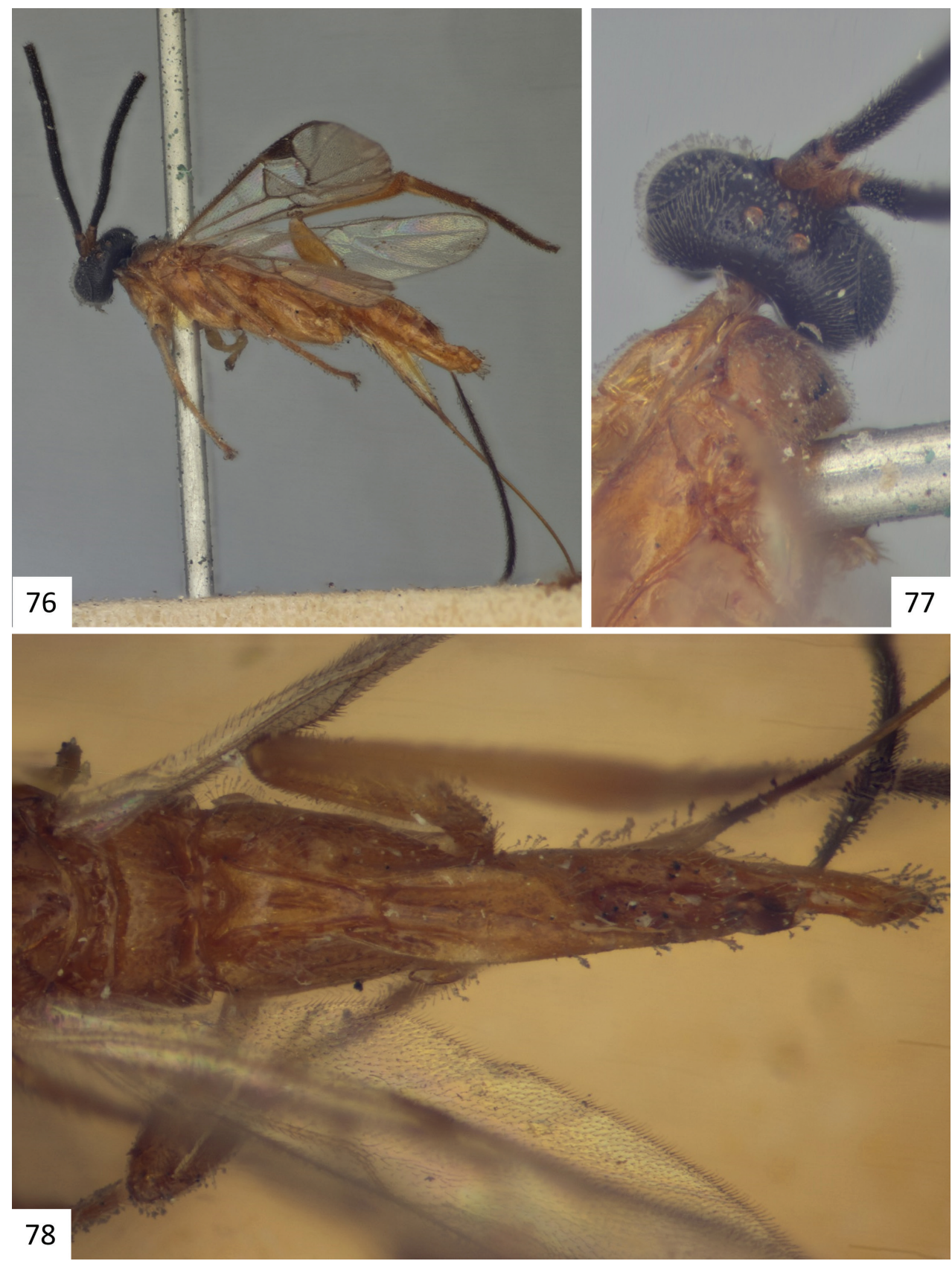

Figures 76-78. Sendaphne sulmo, female holotype. 76 Habitus, lateral view 77 Head and mesosoma (partially), dorsal view 78 Propodeum and metasoma, dorsal view. 

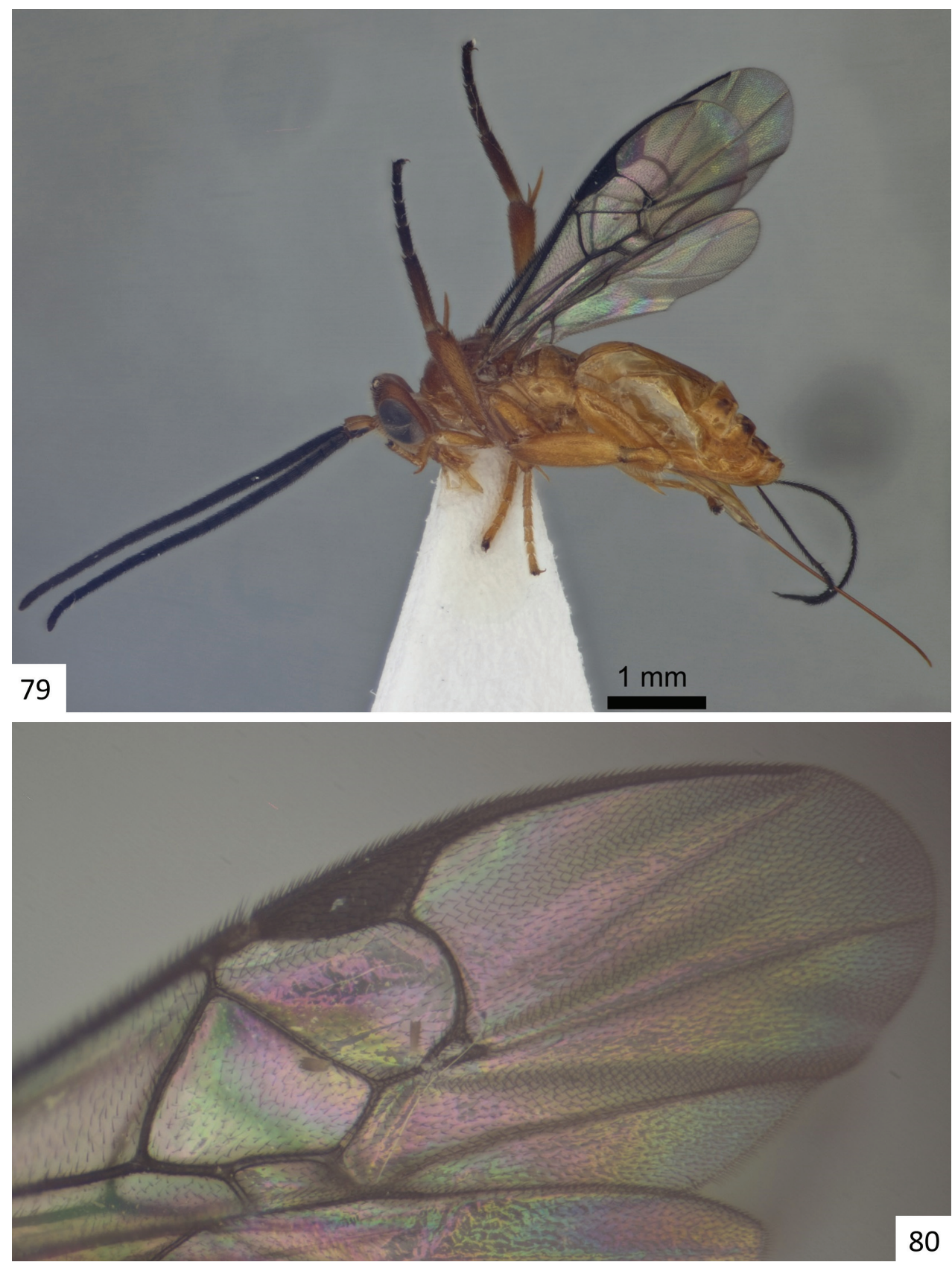

Figures 79-80. Sendaphne olearus, female specimen from Peru. 79 Habitus, lateral view 80 Detail of the fore wing. 
Sendaphne sulmo Nixon, 1965: 204.

Figs 8-14, 76-78

Holotype. Female, BMNH. MEXICO, Tabasco, Teapa (not examined).

Specimens examined. $3 \hat{\circ}$ (CNC). Mexico, Chiapas, $16^{\circ} 58^{\prime} \mathrm{N}, 91^{\circ} 47^{\prime} \mathrm{W}, 560 \mathrm{~m}$; (collecting dates: 6.ix.1978, 28.x.1978, 8-11.xi.1978); coll. J. Rawlins. DNA Voucher codes: CNCHYM 07030 CNCHYM 07031 and CNCHYM 07033.

Diagnosis. This is the only known species of Sendaphne with a higher ocellar triangle (i.e., anatomical line tangent to posterior margin of anterior ocellus crosses far above anterior margin of posterior ocelli). The distance between anatomical line tangent to posterior margin of anterior ocellus and anterior margin of posterior ocelli is $0.5 \times$ the diameter of anterior ocelli (Fig. 77), while for all other known species of Sendaphne it is usually $0.1-0.3 \times$.

Distribution. Only known from lowlands (100-560 m) of Mexico.

Comments. We could only study some photos of the holotype (Figs 76-78) and the original description which included a line drawing of the metasoma (Nixon 1965: 207 , Figure 255). The drawing shows a T1 slightly narrower medially than the photos of the actual holotype reveal, but the rest of the original description is in agreement with the photos we examined. The males mentioned above are, however, different from the female holotype in having a darker anteromesoscutum, scutellar disc, and metasoma (Figs 8-14), and also the ocellar triangle is not as elevated. Lacking more specimens to examine (especially females), we have refrained from considering those male specimens as a different species because the two localities are not too far apart and males are known to be darker in other species of Sendaphne. If more material becomes available for study in the future, the status of those specimens may be clarified.

Molecular data. The three male specimens sampled for DNA rendered minibarcodes of 103 base pairs each.

\section{Acknowledgements}

We emphatically and gratefully acknowledge the support of the ACG parataxonomist team in finding and rearing these caterpillars, their parasitoids and their hyperparasitoids, and Area de Conservacion Guanacaste (ACG) for preserving the forests in which they live, and the Guanacaste Dry Forest Conservation Fund, the Wege Foundation, the International Conservation Fund of Canada, the JRS Biodiversity Foundation, Jessie Hill, Steve Stroud, Permian Global, and the University of Pennsylvania for funding portions of the research. Several Colombian specimens were collected during a project supported by NSF grant DEB 0205982 awarded to M. J. Sharkey and B. V Brown. This study was also supported by NSF DEB 0515699 to DHJ and by a Natural Sciences and Engineering Research Council of Canada (NSERC) Discovery Grant to MAS. Laboratory analyses of these sequences were funded by the Government of Canada through Genome Canada and the Ontario Genomics Institute (2008-0GI- 
ICI-03), and by BOLD/iBOL of the Biodiversity Institute of Ontario and University of Guelph. JFT thanks Yves Braet (Belgium) for making specimens from French Guiana available for this study and Gavin Broad (BMNH) for sending pictures of the specimens housed in London. The suggestions from two anonymous reviewers and the editor considerably improved the final version of this paper.

\section{References}

Braet Y (2006) Coup doeil sur la biodiversité des Braconidae (Hymenoptera, Ichneumonoidea) de Guyane francaise et reclassication de certaines espèces décrites par Brullé. Bulletin de la Société Royale Belge d'Entomologie 142(7-12):155-172.

Fernández-Triana J, Whitfield JB, Rodriguez JJ, Smith MA, Janzen DH, Hallwachs W, Hajibabaei M, Burns JM, Solis MA, Brown J, Cardinal S, Goulet H, Hebert PDN (2014) Review of Apanteles sensu stricto (Hymenoptera: Braconidae, Microgastrinae) from Area de Conservación Guanacaste, northwestern Costa Rica, with keys to all described species from Mesoamerica. Zookeys 383: 1-565. doi: 10.3897/zookeys.383.6418

Hajibabaei M, Smith MA, Janzen DH, Rodriguez JJ, Whitfield JB, Hebert PDN (2006) Identifying specimens with degraded DNA using minimalist barcodes. Molecular Ecology Notes 6: 959-964. doi: 10.1111/j.1471-8286.2006.01470.x

Hebert PDN, Cywinska A, Ball SL, deWaard JR (2003) Biological identifications through DNA barcodes. Proceedings of the Royal Society B 270: 313-321. doi: 10.1098/rspb.2002.2218 Huber JT, Sharkey MJ (1993) Structure. In: Goulet H, Huber JT (Eds) Hymenoptera of the world: an identification guide to families. Agriculture Canada Research Branch, Monograph No. 1894E, Ottawa, Canada, 13-59.

Ivanova NV, deWaard JR, Hebert PDN (2006) An inexpensive, automation-friendly protocol for recovering high-quality DNA. Molecular Ecology Notes 6: 998-1002. doi: 10.1111/j.1471-8286.2006.01428.x

Karlsson D, Ronquist F (2012) Skeletal morphology of Opius dissitus and Biosteres carbonarius (Hymenoptera: Braconidae), with a discussion of terminology. PLoS ONE 7(4): e32573. doi: 10.1371/journal.pone.0032573

Mason WRM (1981) The polyphyletic nature of Apanteles Foerster (Hymenoptera: Braconidae): A phylogeny and reclassification of Microgastrinae. Memoirs of the Entomological Society of Canada, Ottawa, Canada, 147 pp.

Nixon G (1965) A reclassification of the tribe Microgasterini (Hymenoptera: Braconidae). Bulletin of the British Museum (Natural History), Entomology series, Supplement 2: 1-284.

Penteado-Dias AM (1995) Duas espécies novas de Sendaphne Nixon (Hymenoptera, Braconidae, Microgastrinae) do Brasil. Revista Brasileira de Zoologia 12(2): 251-254. doi: 10.1590/S0101-81751995000200004

Ratnasingham S, Hebert PDN (2007) BOLD: The Barcode of Life Data System (www.barcodinglife.org). Molecular Ecology Notes 7: 355-364. doi: 10.1111/j.1471-8286.2007.01678.x

Scatolini D, Penteado-Dias AM (1999) A new species of Sendaphne (Hymenoptera, Braconidae) from Brazil. Entomological News 110(1): 53-55. 
Smith MA, Woodley NE, Janzen DH, Hallwachs W, Hebert PDN (2006) DNA barcodes reveal cryptic host-specificity within the presumed polyphagous members of a genus of parasitoid flies (Diptera: Tachinidae). Proceedings of the National Academy of Sciences 103: 3657-3662. doi: 10.1073/pnas.0511318103

Smith MA, Wood DM, Janzen DH, Hallwachs W, Hebert PDN (2007) DNA barcodes affirm that 16 species of apparently generalist tropical parasitoid flies (Diptera, Tachinidae) are not all generalists. Proceedings of the National Academy of Sciences 104: 4967-4972. doi: 10.1073/pnas.0700050104

Smith MA, Rodriguez JJ, Whitfield JB, Deans AR, Janzen DH, Hallwachs W, Hebert PDN (2008) Extreme diversity of tropical parasitoid wasps exposed by iterative integration of natural history, DNA barcoding, morphology, and collections. Proceedings of the National Academy of Sciences 105: 12359-12364. doi: 10.1073/pnas.0805319105

Smith MA, Bertrand C, Crosby K, Eveleigh ES, Fernandez-Triana J, et al. (2012) Wolbachia and DNA Barcoding Insects: Patterns, Potential, and Problems. PLoS ONE 7(5): e36514. doi: 10.1371/journal.pone.0036514

Whitfield JB (1997) Subfamily Microgastrinae. In: Wharton RA, Marsh PM, Sharkey MJ (Eds) Manual of the New World genera of the family Braconidae (Hymenoptera). Special Publication No. 1. International Society of Hymenopterists, Washington, D.C., 333-364. 


\section{Supplementary material I}

\section{Details of Sendaphne specimens with sequences in BOLD.}

Authors: Jose L. Fernandez-Triana, James B. Whitfield, M. Alex Smith, Winnie Hallwachs, Daniel H. Janzen

Data type: species data

Explanation note: The Excel file contains details on locality, sampling date, collectors, museum codes, sequence length, and other data related to Sendaphne specimens.

Copyright notice: This dataset is made available under the Open Database License (http://opendatacommons.org/licenses/odbl/1.0/). The Open Database License $(\mathrm{ODbL})$ is a license agreement intended to allow users to freely share, modify, and use this Dataset while maintaining this same freedom for others, provided that the original source and author(s) are credited.

\section{Supplementary material 2}

\section{Data for phylogenetic analysis.}

Authors: Jose L. Fernandez-Triana, James B. Whitfield, M. Alex Smith, Winnie Hallwachs, Daniel H. Janzen

Data type: sequence data

Explanation note: Neighbor-Joining tree based on Kimura 2-parameter distances of all described species of Sendaphne with DNA barcodes available. Sequence data from the Barcode of Life Data Systems (http://www.boldsystems.org/). For every sequence the species name, specimen code, length of sequence (in base pairs), and country/province or country/state is shown.

Copyright notice: This dataset is made available under the Open Database License (http://opendatacommons.org/licenses/odbl/1.0/). The Open Database License $(\mathrm{ODbL})$ is a license agreement intended to allow users to freely share, modify, and use this Dataset while maintaining this same freedom for others, provided that the original source and author(s) are credited. 